\title{
Reversible Freeze-Induced Beta-Sheet-to-Disorder Transition in Aggregated Homopolypeptide System
}

Matylda Wacławska a, Marcin Guza a, Grzegorz Ścibisza, Mateusz Fortunka a, Robert Dec ${ }^{a}$, Wojciech Puławski ${ }^{b}$ and Wojciech Dzwolak ${ }^{a^{*}}$

a Faculty of Chemistry, Biological and Chemical Research Centre, University of Warsaw, 1 Pasteur Str., 02-093 Warsaw, Poland. ${ }^{b}$ Institute of High Pressure Physics, Polish Academy of Sciences, Sokołowska 29/37 Str., Warsaw, 01-142 Poland

\section{Supporting Information for Publication}

\author{
Auxiliary IR and AFM data
}

1. Dependence of $\beta$-sheet content in PLL-PLGA aggregate on the stoichiometry of mixing.

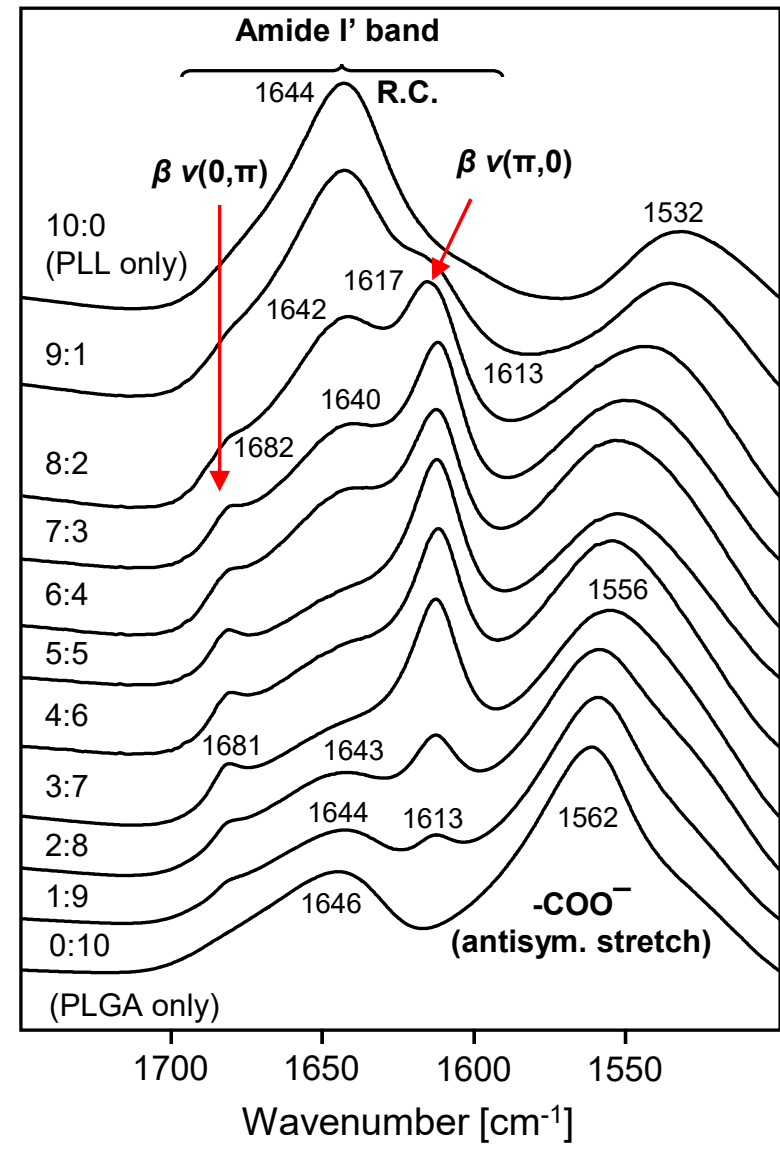

FIGURE S1: Infrared spectra (ATR-FT-IR) of dry films obtained by evaporation of liquid samples prepared by $24 \mathrm{~h}$-long mixing of 1 wt. \% solutions of PLL (hydrobromide salt) and PLGA (sodium salt) in $\mathrm{D}_{2} \mathrm{O}$ at various molar ratios of Lys and Glu side chains, as specified. Pure salts are in the disordered (R.C.) conformation which is reflected by the amide I' band position around $1644-1646 \mathrm{~cm}^{-1}$. Mixing of PLL and PLGA enables Coulombic interactions between both polypeptides resulting in the precipitation of antiparallel $\beta$ sheet (the two exciton-split components at ca. 1682 and $1613-1617 \mathrm{~cm}^{-1}$ corresponding to this conformation are indicated with red arrows). The R.C. $\rightarrow \beta$-sheet transition is most effective for the equimolar mixing ratio. 
2. High frequency regions of the infrared spectra of PLL-PLGA films.

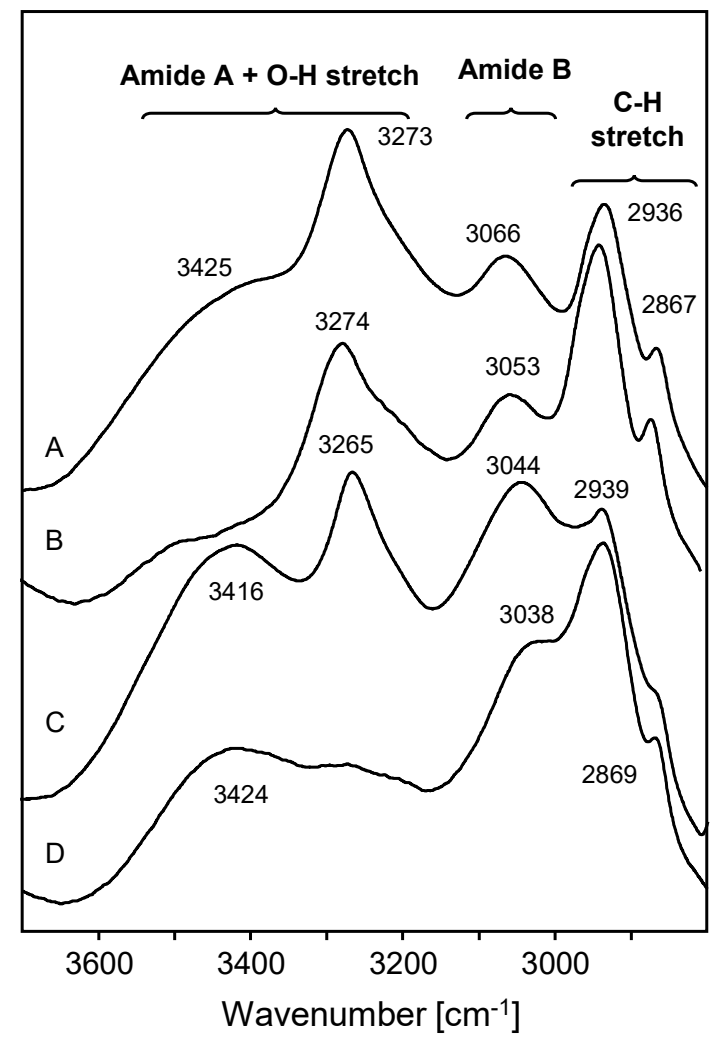

FIGURE S2: High frequency regions of the infrared (ATR-FT-IR) spectra of dry films of PLLPLGA aggregates shown in Fig. 1 (equimolar in terms of Lys and Glu side chains ratio aggregates formed in $\mathrm{H}_{2} \mathrm{O}$ in presence or absence of $\mathrm{NaBr}$ and dried afterwards). Traces of residual $\mathrm{NaBr}$ were removed from samples $\mathrm{A}$ and $B$, whereas additional amount of $\mathrm{NaBr}(0.7 \mathrm{M})$ was added to samples $C$ and $D$ prior to drying. Samples $A$ and $C$ were dried with a stream of air, samples $B$ and $D$ were freeze-dried. Comment: conformational disorder manifests in the disappearance of the sharp $\sim 3270 \mathrm{~cm}^{-1}$ peak (D). The overall intensity decrease above $3100 \mathrm{~cm}^{-1}$ versus $\mathrm{C}-\mathrm{H}$ stretching bands suggests that the freeze-dried samples contain less residual water.

\section{Control experiment: no $\beta \rightarrow$ disorder transition is observed in the absence of salt.}

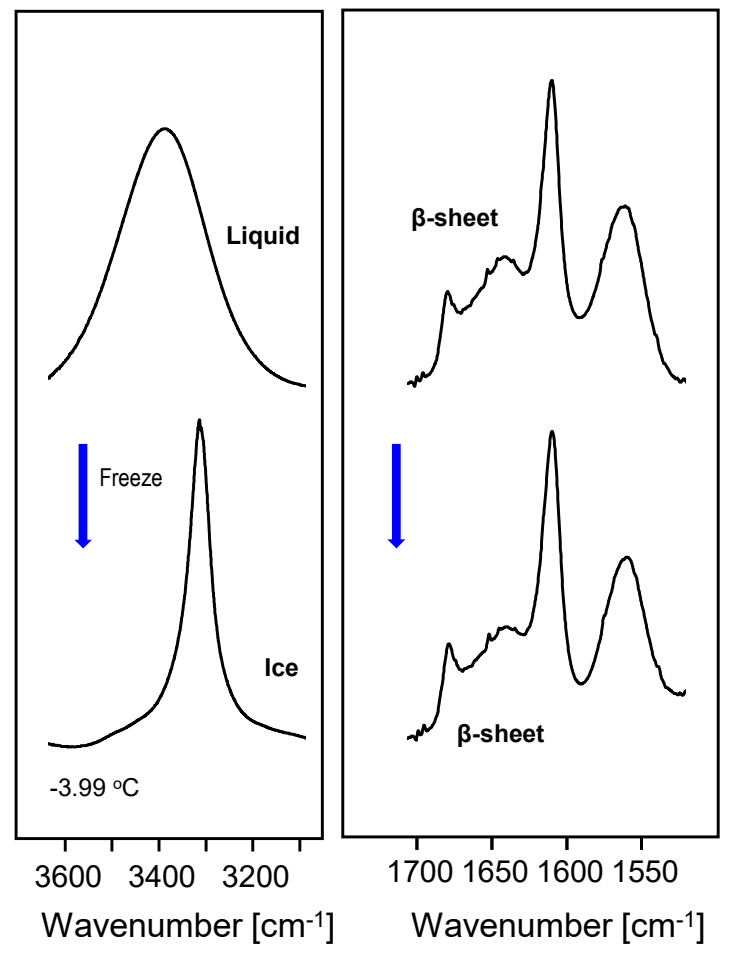

FIGURE S3: Freezing of 2 wt. \% suspension of 1:1 PLL-PLGA aggregate in $\mathrm{D}_{2} \mathrm{O}$ in the absence of $\mathrm{NaBr}$ does not induce the $\beta$-sheet $\rightarrow$ disorder transition. Solvent/ice-subtracted spectra of aggregate before (upper) and after (bottom) freezing in the amide l' band region (right panel); the corresponding region of $\mathrm{O}-\mathrm{H}$ stretching vibrations visible in unsubtracted spectra (left panel). 
4. Freeze-thaw cycles.

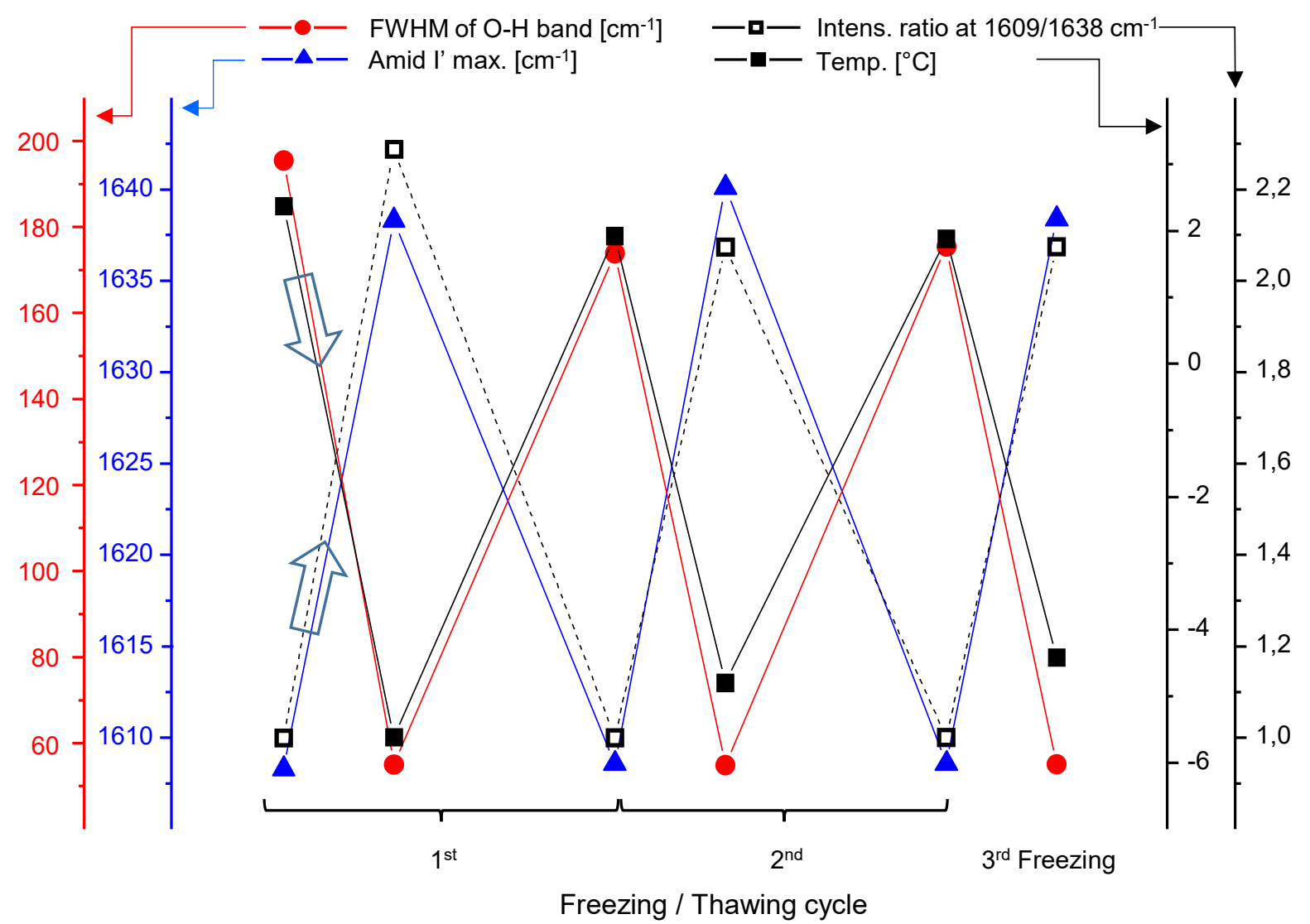

FIGURE S4: Reversibility of the $\beta$-sheet $\rightarrow$ disorder transition upon consecutive cycles of freezing/thawing (2 wt. \% suspension of 1:1 PLL-PLGA aggregate in $0.7 \mathrm{M} \mathrm{NaBr}$ in $\mathrm{D}_{2} \mathrm{O}$ ).

\section{AFM images of PLL-PLGA aggregates freeze-dried in the presence of $\mathrm{NaBr}$.}

PLL-PLGA ionic aggregates are amorphous. The freeze-induced transition in the presence of $\mathrm{NaBr}$ does not appear to affect this property (morphology of this system is challenging to probe using either transmission electron microscopy (TEM) with negative staining or atomic force microscopy (AFM)). Samples of aqueous suspensions of PLL-PLGA aggregates (1:1 Lys:Glu side chain ratio) were diluted 40 times with water or $0.2 \mathrm{M} / 0.7 \mathrm{M}$ aqueous $\mathrm{NaBr}$ solutions. Small portions of thus prepared liquid samples were swiftly deposited onto freshly cleaved mica and subjected to in situ overnight freeze-drying. AFM tapping-mode measurements were carried out using a Nanoscope III atomic force microscope from Veeco, U.S.A., and TAP300-Al sensors (res. frequency $300 \mathrm{kHz}$ ) from BudgetSensors, Bulgaria. The cross-sections of selected specimen were obtained from the corresponding height images. 

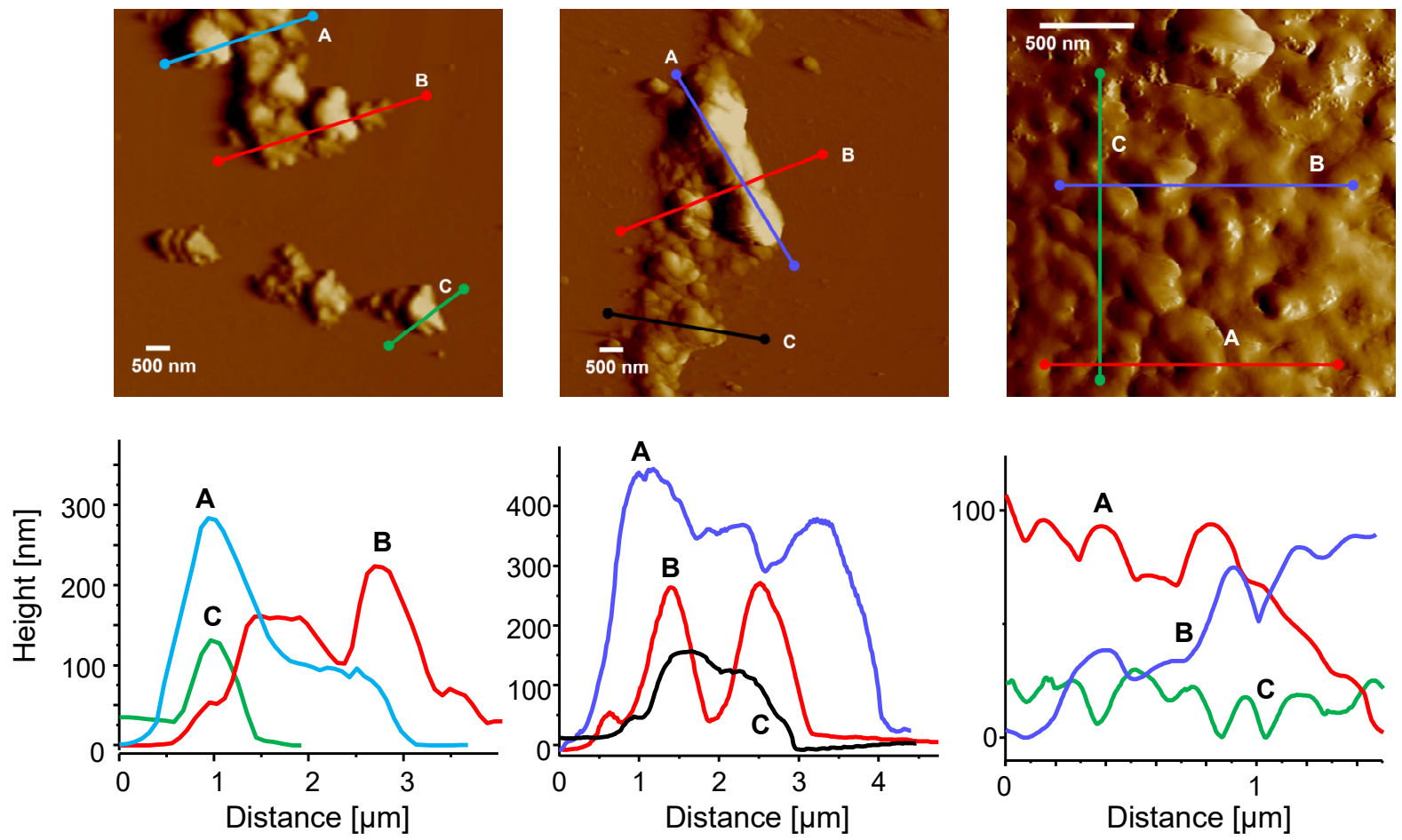

FIGURE S5: AFM amplitude images (upper row) of 1 wt. \% PLL-PLGA aggregates freezedried in the absence of $\mathrm{NaBr}$ (left column), in the presence of $0.2 \mathrm{M} \mathrm{NaBr}$ (middle column), and in the presence of $0.7 \mathrm{M} \mathrm{NaBr}$ (right column). The indicated cross-sections of selected specimen (bottom row) were obtained from the corresponding height images. Comment: the aggregates are amorphous and lack characteristic morphological features that could be tracked upon the transition. In the presence of high concentrations of $\mathrm{NaBr}$, freeze-dried PLL-PLGA remains strongly associated. The AFM data shows no evidence of morphological changes occurring upon the transition that could be attributed solely to the conformational transition itself. Presence of $\mathrm{NaBr}$ microcrystals forming during the freezing of samples in 0.2 $\mathrm{M} / 0.7 \mathrm{M}$ solutions of the salt poses an additional challenge to morphological analysis. 